\title{
Wellbeing in Education: Staff Matter
}

\author{
Nadia Lovett and Trevor Lovett
}

\begin{abstract}
This paper discusses the development and preliminary findings of a pilot study concerning the wellbeing of staff at a senior secondary school in Adelaide, South Australia. Ten respondents, randomly selected from the staff of over fifty individuals, were invited to participate in the study "Wellbeing (a) work". Respondents rated their wellbeing using the PERMA Profiler. PERMA is a theory that positions wellbeing according to five domains $P$ (positive emotion), $E$ (engagement), $R$ (positive relationships), $M$ (meaning) and $A$ (accomplishment). In addition to the PERMA Profiler were three qualitative wellbeing questions. The aim of using school staff members to measure wellbeing has a twofold purpose: to add to the existing literature on wellbeing and to illustrate that we measure what we treasure and what we measure affects what we do. Measuring the wellbeing of those who work in learning institutions demonstrates that this aspect of their lives is important. The data from the survey will provide respondents a medium through which their strengths as well as areas for growth can be monitored. The findings of the study will not only provide individuals with a snapshot of wellbeing but also indicate the direction for whole staff initiatives that can promote flourishing.
\end{abstract}

Index Terms-Measurement, positive education, school, staff, wellbeing.

\section{INTRODUCTION}

Psychology has traditionally focussed on illness and dysfunctional behavior [1]. However the study of positive psychology became the area of worldwide research after Professor Martin Seligman was appointed president of the American Psychological Association in 1997 [2]. Positive psychology focuses more on what is going well in a person's life and what makes life worth living [3] in contrast to directing attention to illness and deficits. It is a scientific study that explores how individuals, groups and communities use their strengths, characteristics and purposeful actions to live happy, healthy and meaningful lives [4]. Positive psychology complements rather than replaces traditional forms of psychology. Interventions targeted to alleviate mental illness will continue to exist, however positive psychology uses strength-based universal interventions that develop individual and large-scale emotional, social and physical capital [3].

Recent research has revealed the nexus between an individual's wellbeing and learning [5]. Wellbeing before learning was the emphasis of the Adelaide Thinkers in

Manuscript received July 9, 2014; revised October 15, 2014.

N. M. Lovett is with the University Senior College, at The University of Adelaide, South Australia, Australia (e-mail: nadia.lovett@adelaide.edu.au ).

T. W. Lovett is with the School of Education, University of South Australia, Australia (e-mail: Trevor.Lovett@unisa.edu.au ).
Residence program in 2012-2013 [6]. (The Thinker in Residence program was initiated in South Australia at the beginning of 2003. This innovative program encouraged global leaders from a diversity of fields to collaborate with local and state-wide communities and develop strategies to put new ideas into practice. The principal outcome of the programs was to improve the lives of South Australians [7].) In 2012 and 2013 the Adelaide Thinker was renowned psychologist and world leader in wellbeing, Professor Martin Seligman.

Professor Seligman and partners in the Thinkers' program encouraged leaders in South Australian schools and other organisations to lead, measure and build wellbeing within their communities [6]. We measure what we treasure [8] was often cited during Seligman's residency. It seems logical that learning institutions measure the academic achievement and wellbeing of students. This paper adds to the body of knowledge by measuring the wellbeing of teachers and ancillary staff members who are influential in the lives of students. By gathering baseline data, assessment of staff wellbeing can be developed through self-monitoring, and the teaching and learning of evidence-based wellbeing interventions. As a consequence the progress of the effects of such interventions can be evaluated [9], [10].

National data regarding the frequency of mental health issues in adults in the workplace, and the costs related to those issues are important because they identify the importance of measuring the wellbeing of adults. According to Australian research around twenty percent of adults at any one time experience a mental health condition [11], and approximately forty-five percent of adults living in Australia will experience a mental health condition during their lifetime [12]. Anxiety and depression are experienced by more than three million Australians [13]. Absenteeism, loss of productivity and insurance claims from depression and anxiety-based issues are said to cost the Australian economy over $\$ 10.1$ billion annually [14].

Data related to Australian teachers' wellbeing indicate fifty percent of teacher illness is stress-related: twice the national workforce average. The retention rate of newly appointed teachers, following five years of service, is around sixty percent. Approximately forty percent of teacher recruits reveal stress as one of the primary reasons for leaving the teaching profession. Of all occupations, secondary school teachers are second only to police in relation to the most stress-related Workcover claims [15].

Since 2006 a number of research studies regarding employee happiness have been conducted. The findings suggest that many employers are investing time and money in the measurement and building of employee wellbeing. Employee happiness has been linked with staff retention and productivity. Organizations worldwide, including Google, 
Etzy and the Bank of America are keen to learn how to attract, develop and retain employees who are happy. Collecting baseline data through the measurement of happiness and wellbeing is considered an important first step. Caring about worker happiness and wellbeing has a tipping effect: it benefits the employee, the productivity of the organization and the community at large [16].

The Australian statistics, regarding the prevalence and costs of mental ill-health, teacher retention as well as research into happy and healthy organizations, demonstrate not only the moral but also the economic sense of making the health and wellbeing of school staff a priority. Educational institutions do have existing suites of policies and practices for staff that address health and safety matters, for example the Work health, safety and injury management policy [17]. Nonetheless there is little evidence that regular state-wide educational institutional specific data pertaining to staff wellbeing are collected and used to inform policies and practices.

The main objective of Martin Seligman's, Thinker in Residence Program for 2012-2013, was to develop South Australia into a flourishing state. The broad and comprehensive method for achieving this objective was a cyclical approach based on three principles of: leading, measuring, and building the state in ways that improve individual and collective wellbeing enabling the population to thrive. The method is considered to be both practical and accessible to all South Australians where 'everyone has a role to play in wellbeing' [9]. The aforementioned three principles inform the research on which this paper is based.

While a number of theories explain the structure of wellbeing [18], [19], PERMA, Seligman's 2011 theory of wellbeing, is the one used in this research. Seligman's PERMA theory has five measurable and teachable domains. The acronym PERMA stands for $\mathrm{P}$ (positive emotion), $\mathrm{E}$ (engagement), $\mathrm{R}$ (positive relationships), $\mathrm{M}$ (meaning) and $\mathrm{A}$ (accomplishment). These domains can be defined and measured separately. Below are brief descriptions of each of the five domains.

\section{A. Positive Emotion}

The value of positive emotion for wellbeing has been well researched [20]. Although it has been documented that individuals can experience positive and negative emotions at the same time and that negative emotion can be both appropriate and helpful [1] (as in expressing grief after a loss, for example) evidence reveals that genuine regular expressions of positive emotion and engagement in activities that induce positivity help individuals to flourish [21]. Positive emotions help to broaden one's awareness and help to build resources. They allow people to develop an open mindset, which in turn builds social and emotional capital. Although positive emotions can be short lived their impact can be long lasting. The frequency of positive emotions can help to counteract the effects of negative emotion [22]. Fredrickson and Losada identified that an individual's positivity ratio can be used to determine their quality of life. A 3:1 ratio of positive to negative emotion is regarded as a tipping point. Individuals who experience this level of positive emotion in relation to their negative emotion are seen to be thriving [21], [23].

\section{B. Engagement}

The term engagement has also been referred to as flow; when an individual is so engrossed in activity they lose the sense of time and sense of self [24]. Any activity can induce flow. The activities do not always need to be challenging for flow to be experienced [10]. According to Csikszentmihayli, a good life can be defined by living in a constant state of flow where one is totally absorbed and engaged in the present [25]. Generally thoughts and emotions are not generated when an individual is in flow however on reflection of the activity; the person indicates that the experience has generated positive emotion [26]. Flow can produce a natural high, however, once those activities, that originally produced the high are mastered, the natural high tends to dissipate. In order for flow to be maintained, activities need to have a degree of challenge [10]. Studies show that flow and engagement in the workplace are often referred to as energy, dedication and absorption in the tasks at hand [27].

\section{Relationships}

Research about the importance of positive relationships to mental health and wellbeing is well documented. The number of relationships, the perceived quality of such relationships and the value of support received and given to others has been measured in many global studies [28]-[30]. The quality of relationships is seen to be essential for our own level of happiness [31]. Evidence suggests that positive relationships are antidotes to the low moments in one's life and buffers for depression and loneliness [32]. It is often in the company of others that individuals experience positive emotion. Doing something for someone else, as in engaging in an act of kindness, has been proven to increase levels of positive emotion, which in turn potentially increases the wellbeing of both the giver and the receiver [10].

\section{Meaning}

Meaning provides us with an understanding of who we are and with which group we belong [33]. It is constantly being exchanged and refined through personal reflection and social interactions [34]. Meaning is often referred to when an individual is aware of the purpose of their life. It is when individuals feel they are fulfilled and are engaging in activities that matter. Meaning in life had been studied long before the advent of the positive psychology movement [35]. Meaning is a subjective measure that attributes to wellbeing [26]. Some studies have detailed that at significant moments in people's lives they question what their life is all about. In his memoirs of life in concentration camps during WWII, renowned psychiatrist Viktor Frankl, asked his readers to consider what meaning they can give to life itself rather than expecting life to provide them with meaning. Finding meaning in life is often seen as part of life's journey. The capacity to make sense of life's experiences may provide individuals with hope for the future. In Frankl's WWII memoirs, meaning is possibly found in work, in caring and connecting with others and in the courage of dealing with life's challenges [36]. Even though having a meaningful life can evoke positive emotion, dealing with the struggles of one's life is the way people find meaning [35]. 


\section{E. Accomplishment}

Accomplishment is also referred to as achievement. It is often objectively measured and pursued even if it doesn't induce positive emotion or meaning. Accomplishment can also be measured subjectively and has been added to the wellbeing domain as it can involve defining and working towards goals, which can result in mastering specific skills and developing self-efficacy [28].

Seligman and the partners in the Thinkers in Residence 2012-2013 program encouraged leaders in institutions, including schools, to use the scientifically-based rubric of PERMA to measure wellbeing. Leaders are urged to gather baseline data to assess wellbeing and have that data inform the building, teaching and learning of PERMA through regular application of intentional wellbeing principles. The progress and evaluation of the wellbeing interventions are to be measured on a regular basis. Leading the way through sharing the measuring and building of PERMA is also encouraged, as it will promote discussion, reflection and action in the area [9].

\section{METHOD}

Respondents were employees of an independent, coeducational, senior secondary school in Adelaide. One of the researchers is the school counsellor. Twelve individuals were randomly selected from the staff of over fifty. The sample consisted of nine teaching staff (including three leaders) and three ancillary staff. Three of the twelve were males. An email was sent to the prospective participants inviting them to participate in the voluntary and anonymous pilot study. Of the twelve, ten chose to participate.

The PERMA Profiler was chosen as the subjective wellbeing measurement tool to learn more about employees' wellbeing. The measure not only fitted the vision and plan of Seligman's residency but was also adopted as a consequence of the school counselor attending a measurement master class, delivered by Dr Margaret (Peggy) Kern, one of the co-developers of the PERMA Profiler, in early 2014 [37].

Following the measurement conference the counselor has both met and corresponded with Dr Kern in relation to measuring staff wellbeing and trailing the PERMA Profiler with the staff at the senior secondary school. Dr Kern's permission to use the PERMA Profiler within the school setting was granted. Approval to conduct the pilot study was given by the principal after discussions with school leadership and subsequent sharing of current research in staff wellbeing and the use of the PERMA Profiler.

The PERMA Profiler is a brief 23 item measure that includes three questions for each of the five wellbeing constructs in addition to questions about negative effect, physical health and loneliness. Respondents are asked to answer the questions using an eleven point Likert scale. The measure's reliability has been established by its application to approximately 12,000 participants worldwide. On completion of the measure a profile of each of the domains is created [28].

Respondents to the pilot study were emailed a link to the online survey and asked to complete it within the week. The survey consisted of the PERMA Profiler's quantitative questions and three additional qualitative questions. The qualitative responses related to an individual: defining wellbeing, listing what they do to enhance their wellbeing and providing feedback on the survey. The aim of the three additional questions was to: make necessary improvements to the survey, incorporate staff views in a wellbeing policy and ultimately open discussion about wellbeing practices in the hope that staff may ultimately consider and adopt additional or alternate activities that enhance wellbeing.

Ethical protocols were followed to ensure the anonymity and welfare of the respondents was maintained. On completion of the survey, respondents were asked to copy and keep their results. They were given instructions to calculate means for the various domains which then produced their personalized PERMA profile. Respondents were informed that they would be sent another link in a fortnight's time to complete the survey again. By using the PERMA Profiler to obtain baseline data, and at a later stage additional data, it is hoped that individuals will monitor their wellbeing, acknowledging areas in which they are doing well and those requiring growth so as to increase their levels of flourishing.

\section{FINDINGS AND DISCUSSION}

The data gathered by the survey were initially collated using the online professional software tool, Surveygizmo [38]. Summary pie graphs were created for each of the PERMA Profiler questions but after discussion with Dr Kern it was suggested that an alternate data display was more appropriate. There were three items for each of the PERMA constructs (totalling 15) and three items related to negative affect (anger, sadness and anxiety), three items assessing physical health (assessment of health, satisfaction of health and comparison of health with others) and a single item to assess loneliness. The mean of each item (except for the single item, loneliness) was calculated and an excel spreadsheet a PERMA profile depicting a snapshot of each participant's wellbeing was created. Fig. 1 depicts one of the respondent's profiles. Fig. 2 is a graph depicting the collective PERMA profile.

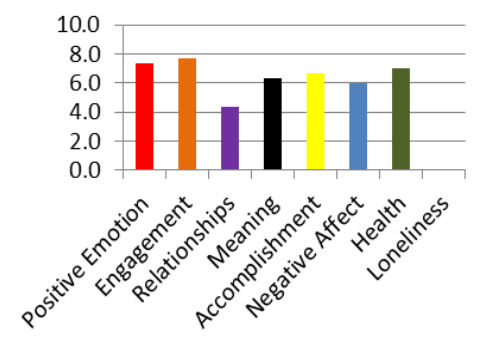

Fig. 1. An example of a participant's PERMA profile.

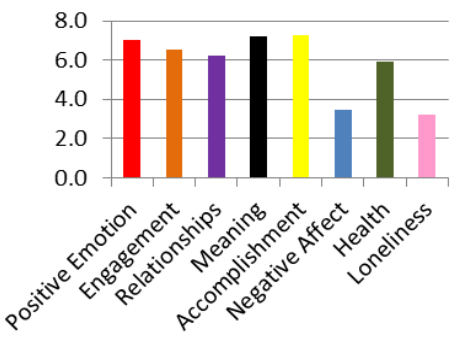

Fig. 2. Collective PERMA profile. 
A collective profile would be useful for future discussions with staff in the workplace. Discussion of how the data can be interpreted and what would need to occur to increase levels of flourishing in the staff may be posed. Reflecting on the collective PERMA Profiler which provided the mean for each of the five PERMA domains, including Negative effect, Health and Loneliness, showed a range of scores from 6.2 out of 10 (positive relationships) to a score of 7.3 out of a possible 10 (accomplishment).

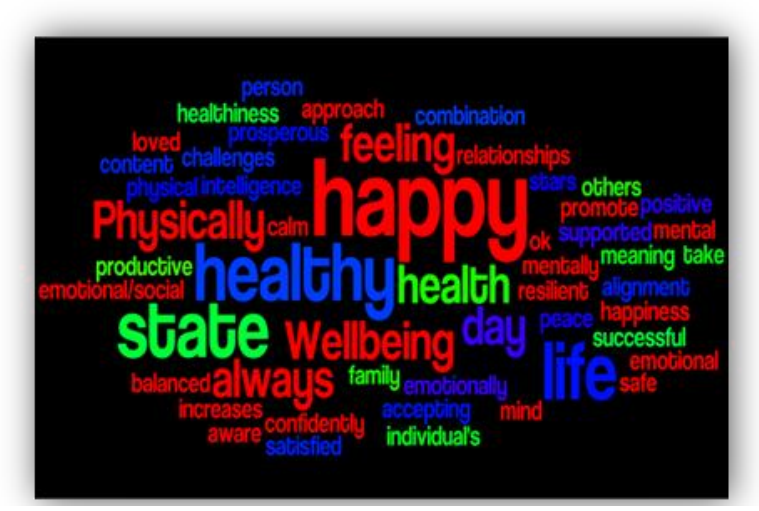

Fig. 3. A word cloud created with wordle. It depicts the frequency of words used by participants to define wellbeing.

The data (see Fig. 3), generated from the first qualitative question Please provide your definition of wellbeing? were first collated using the Wordle [39] online program for generating word clouds. Wordle provides a cloud of the frequency of words used in the definition. Before entering the words on the website those that had no relevance were deleted, such as etc, and, for example. The most frequently used words are noted by size. For example the word happy was a commonly used word among the ten respondents, so the size of the word was large, whereas words like calm, ok, and challenges, were infrequently used and are therefore written in the smallest font.

The definitions were also analysed using the five PERMA domains including the physical health, negative affect and loneliness constructs of the PERMA Profiler measure. There were thirteen positive emotional words in the definitions, resulting in more terms dealing with the $\mathrm{P}$ (positive emotion) domain than any of the others. In contrast, words for the other four domains were minimal (E1, R2, M2, A1). The term physical health was included in the wellbeing definitions of three respondents. In two definitions the terms mental health and emotional health were mentioned.

It is envisaged that the definition data from the pilot study would be highlighted and discussed during staff and wellbeing committee meetings. The data would be used to inform the sections of a future staff wellbeing policy. This would help to make the policy inclusive of staff voices and is expected to result in their ownership of the policy. Inclusivity and being heard are recognised as aspects that help create a mentally healthy workplace [40].

The second qualitative question, What do you do to enhance your wellbeing? was also analysed using the PERMA constructs. The data were congruent with worldwide studies that others matter [3]. There were twelve references to the importance of relationships. Quality relationships with family, friends, colleagues and even the pet dog were significant to eight of the ten respondents. This again matches other research findings that relationships with others, including pets [41] are vital in people's lives and enhance wellbeing. Some respondent comments include:

\section{I spend time with family and friends.}

I have lunch in the staffroom 4-5 days a week with my colleagues.

\section{I spend time with family...I walk the dog.}

One participant commented:

I try to avoid those who are negative around me in the workplace.

While this comment refers to relationships it also fits the domain of Positive Emotion. The comment is consistent with work by Fredrickson and Losada about being positive and increasing one's level of positivity [21]. It is viewed that both positivity and negativity have a tipping point. Some believe they are 'contagious'.

Regarding Positive emotion, there were seven references in total. An example is seen below:

I always try to stay positive and happy at work and at home with my family.

It was difficult to ascertain if any of the comments related to the domains of Engagement or Meaning. While one respondent wrote:

\section{I keep my brain active by studying, doing puzzles, etc.}

It is not clear if these activities provide the respondent with sense of flow or engagement or if they provide meaning to their life.

Planning and prioritising can be linked with the domain of Accomplishment through the setting of goals. Two respondents made mention of planning and prioritising as ways to enhance their wellbeing:

\section{I keep on planning and organising my schedule.}

\section{I prioritise work commitments.}

The importance of physical health was mentioned by six of the respondents and there were eight terms that described some form of physical exercise. Examples include:

Yoga, pilates, daily stretching.

\section{Playing sport.}

Other activities that enhance physical health, such as nutrition and sleep for example were not discussed by any respondent.

The final question asked staff to make any comment on the survey. Nine of the ten respondents wrote a comment. Two provided technical feedback, which will inform the layout and administration features of the survey in the future. These included the opportunity to make comment after each question, number of questions per page, issues with completing the survey via a smart phone and the link of the survey being lost mid survey. One individual mentioned,

I felt I was trying to be tricked, by being given pretty much 
the same question again and again. Like you were trying to catch me out on the first answer I gave if I didn't give the same rating next time I answered the question!

This comment was taken into consideration and changes were made to the introduction to the follow-up survey. Two individuals felt their answers might differ depending on their mood at the time or the happenings at work and their workload. While one out of ten respondents felt the survey 'did nothing' for him/her, there were four positive responses including:

\section{Well put together, easy and quick}

\section{Good length}

Wellbeing at work is important. It would be nice to know that you're doing a good job.

It is a good survey, giving me the opportunity to reflect on my job satisfaction and overall feeling of my work/health situation!

The additional three qualitative questions add value to the PERMA Profiler items. They provide opportunities to make further comment on personal understanding of the term wellbeing, what they do to enhance their wellbeing and their reflections on the survey in general.

\section{FUTURE DIRECTION}

To 'complete' the profile participants will be asked to re-sit the survey. Each participant will then have generated two snapshots of their wellbeing. It is envisaged that they compare and contrast their results. It is hoped that the survey would have encouraged some self-monitoring, that would translate into some form of action to improve an original profile. Regardless of the results the data can be presented to the group and to the remainder of the staff during staff meetings. Ideally the whole staff would be involved in creating a personal PERMA profile on an annual basis. Discussion about the results and what else can be done to improve the profiles and the levels of flourishing in the staff is a positive direction for positioning staff wellbeing. Taking the first steps in measuring the wellbeing of staff promotes the message that wellbeing is treasured.

Of value would be for leaders in schools to communicate personally with each member of staff regularly to discuss the individual' $\mathrm{s}$ state of wellbeing and the strategies adopted to promote thriving. Trailing and evaluating some of these activities should not only assist in the flourishing of staff but also help fulfill Professor Seligman's three principles.

\section{REFERENCES}

[1] J. Ciarrochi, T. Kashadan, and R. Harris, "The foundations of flourishing," in Mindfulness, Acceptance and Positive Psychology: The Seven Foundations of Wellbeing, T. Kashadan and J. Ciarrochi eds. Oakland: Context Press. 2013, pp. 1-29.

[2] R. D. Rusk and L. E. Water. (Apr 2013). Tracing the size, reach, impact, and breadth of positive psychology. The Journal of Positive Psychology. [Online]. 8(3). pp. 207-221. Available: http://dx.doi.org/10.1080/17439760.2013.777766
[3] C. Peterson, A primer in Positive Psychology, New York: Oxford, University Press, 2006

[4] S. Gable and J. Haidt, "What (and why) is positive psychology?," Review of General Psychology, vol. 9, no. 2, pp. 103-110, 2005.

[5] Department of Education and Children Services (DECS), DECS Learner Wellbeing Framework from Birth to Year 12, Adelaide: office of Early Childhood and Statewide Services, 2007.

[6] M. Seligman, Building a State of Wellbeing: A Strategy for South Australia, Department of the Premier and Cabinet, Adelaide, 2013

[7] Adelaide Thinkers in Residence. (July 2014). About us. Adelaide Thinkers in Residence. [Online]. Available http://www.thinkers.sa.gov.au/about/default.aspx

[8] J. Stiglitz, A. Sen, and J.-P. Fitoussi. (2009). Report by the Commission on the measurement of economic performance and social progress. The Commission: Paris. [Online]. Available: http://www.stiglitz-sen-fitoussi.fr/en/index.htm

[9] M. Seligman, "Building the state of wellbeing: a strategy for South Australia," A summary of progress, Department of the Premier and Cabinet, Adelaide, 2013

[10] S. Lyubomirsky, The How of Happiness: A Practical Guide to Getting the Life you Want, London: Piatikus, 2007.

[11] P. McGorry, "A promising future for youth mental health," Australian Medical Students Journal, vol. 1, no. 1, pp. 5, 2012,

[12] Heads up. (June 2014). Mental health in the workplace: Key facts. [Online]. Available: http://headsup.org.au/creating-a-mentally-healthy-workplace/mental-h ealth-in-the-workplace

[13] Beyondblue. (May 2014), Resources for managers and leaders. [Online]. Available: http://www.beyonblue.org.au/resources/in-thr-workplace/for-manager s-and-leaders

[14] Safework Australia. (May 2014). Preventing psychological injury under health and safety laws: Fact sheet. [Online]. Available: http://www.safeworkaustralia.gov.au/sites/SWA/about/Publications/D ocuments/855/Preventing-Psychological-Injury-Under-WHS-Laws.pd

[15] Safework Australia. (April 2013). The Incidence of Accepted Workers Compensation Claims for Mental Stress in Australia. [Online]. Available:

http://www.safeworkaustralia.gov.au/sites/SWA/about/Publications/D ocuments/769/The-Incidence-Accepted-WC-Claims-Mental-Stress-A ustralia.pdf

[16] Fast Company. (2013). Why your boss cares if you're happy. Fast Company. [Online]. Available: http://www.fastcoexist.com/3017403/why-your-boss-cares-if-youre-h appy?partner=newsletter

[17] Department of Education and Child Development. (2013), Work health safety and injury management. DECD 2013. [Online]. Available: http://www.decd.sa.gov.au/docs/documents/1WorkHealthSafety.pdf

[18] F. Huppert and T. Ho, "Flourishing across Europe: Application of a new conceptual framework for defining wellbeing," Social Indicators Research, vol. 110, pp. 837-861, 2013.

[19] C. Ryff and C. Keyes, "The structure of psychological wellbeing revisited," Journal of Personality and Social Psychology, vol. 6, pp.719-727, 1995

[20] R. Howell, M Kern, and S. Lyubormirsky, "Health benefits: Meta-analytically determining the impact of wellbeing on objective health outcomes," Health Psychology Review, vol. 13, pp. 1092-1104, March 2007.

[21] B. Fredrickson, Positivity: Groundbreaking Research to Release your Inner Optimist and Thrive, Great Britain: One world Publications. 2011.

[22] E .Garland and B. Fredrickson, "Mindfulness broadens awareness and builds meaning at the attention-emotion interface," in Mindfulness, Acceptance and Positive Psychology: The Seven Foundations of Wellbeing, T. Kashadan and J. Ciarrochi eds., Oakland: Context Press. 2013, pp 30-67.

[23] B. Fredrickson and M. Losada, "Positive affect and complex dynamics of human flourishing," American Psychologist, vol. 60, no. 7, pp. 678-686, 2005.

[24] M. Csikszenrmihakyi and I. Csikszentmihayli, Optimal Experience: Psychological Studies of Flow in Consciousness, Cambridge University Press, 1998

[25] J. Nakamura and M. Csikszentmihalyi, "The concept of flow," in Handbook of Positive Psychology, C. R. Snyder and S. J. Lopez eds., Oxford: Oxford University Press, 2002, pp. 89-205.

[26] M. Seligman, Flourish: A Visionary Understanding of Happiness and Wellbeing, Simon and Schuster, New York, 2011. 
[27] W. Schuaufeli, A. Baker, and Salanova, "The measure of engagement with a short questionnaire: A cross-national study," Educational and Psychological Measurement, vol. 66, pp. 701-716, 2006.

[28] J .Butler and J. Kern, The PERMA-Profiler: a Brief Measure of Flourishing, Philadelphia, Press, PA.

[29] D. Rickwood, F. Deane, C. Wilson, and J. Ciarrochi, "Young people's help seeking for mental health problems," Australian e-Journal for Advancement of Mental Health, Supplement, vol. 4, no. 3, 2005.

[30] C. Wilson and F. Deane, "Adolescent opinions about reducing help-seeking barriers and increasing appropriate help engagement," Journal of Educational and Psychological Consultation, vol. 12, no. 4, pp. 345-346, 2001.

[31] C. Peterson, Pursuing the Goodlife: 100 Reflections on Positive Psychology, Oxford: Oxford University Press, 2013.

[32] R. Baumeister and M. Leary, "The need to belong: desire for interpersonal attachments as a fundamental human motivation," Psychological Bulletin, vol. 117, pp. 497-529, 1995.

[33] K. Woodward, Identity and Difference, London: Sage, the Open University Press, Book 3, 1997.

[34] P. Atkinson and S. Delamont, "Rescuing narrative from qualitative research," in Narrative-State of the Art, M. Bamberg ed. Amsterdam/Philadelphia: John Benjamins Publishing Co. 2007, vol. 6 pp. 195-205.

[35] M. Steiger, K. Sheline, L. Merriman, and T. Kashdan, "Using the science of meaning to invigorate values-congruent, purpose-driven action," in Mindfulness, Acceptance and Positive Psychology: The Seven Foundations of Wellbeing. T. Kashadan and J. Ciarrochi eds., Oakland: Context Press, 2013, pp. 240-266.

[36] V. Frankl, Man's Search for Meaning: the Classic Tribute to Hope from the Holocaust, Great Britain: Random House, 2008.

[37] M. Kern, "The PERMA profiler," presented at the Measuring Wellbeing in Schools: A Masterclass. Seymour College Glen Osmond, Adelaide February 18, 2014.

[38] Surveygizmo. (March 2014). Surveygizmo. [Online]. Available: http://www.surveygizmo.com

[39] J .Feinburg. (2013). Wordle. [Online] Available: http://wordle.net

[40] WC. (May 2014). Creating a mentally healthy workplace: Return on investment analysis. Final Report. Australian Government. [Online] Available: http://www.pwc.com.au

[41] N. Lovett, "Family as helpers: Using digital storytelling to explore the help-seeking behaviour of adolescent girls," presented at Generations of Relationships across Generations Conference. The Combined $7^{\text {th }}$ Annual Conference of the Australian Psychological Society's Psychological of Relationships Interest Group and International Association of Relationship Research Mini-Conference, Australian Catholic University, Melbourne, November 2007.

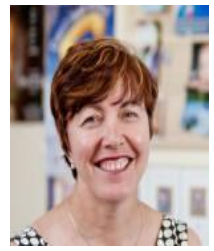

Nadia Lovett was born in Adelaide, South Australia, 1960. She is a member of Counseling Association of South Australia CASA.

She received her doctorate in counselling at the University of South Australia, Adelaide, South Australia, Australia in 2009, master of social science in educational counselling from University of South Australia, Adelaide, Australia in 2000, and graduate diploma in reading and language education from South Australian Collage of the Arts and Education, Adelaide, South Australia, Australia in 1984. She got her diploma in teaching (primary) from Adelaide College of the Arts and Education, Adelaide, South Australia, Australia in 1979 with major in Aboriginal studies and Italian.

Nadia is the school counselor at University Senior College and has been at the college since 2005. She has more than thirty years' experience in working in educational settings as a teacher, school counselor and university lecturer. She has taught students from five years of age to adults in Australia and overseas. In 2009 Nadia completed her doctorate in counseling at the University of South Australia. Her thesis explored the help-seeking behaviors of adolescent girls using digital storytelling as a data gathering tool.

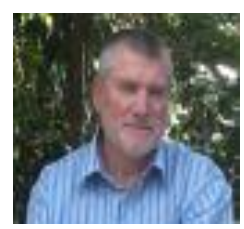

Trevor Lovett was born in Epping United Kingdom 1954. He is a PhD. and member of TASA of the Australian Sociological Association.

He received his $\mathrm{PhD}$ in sociology of education at the University of South Australia, Adelaide, Australia, 2011, master of education at the University of New England, New South Wales, Australia with major in linguistics, 2004, master of letters from University of New England, New South Wales, Italian literature, 2001. He got the certificate of Italian language from Scuola Leonardo da Vinci, Florence, Italy, 2000. He got bachelor of education from South Australian College of Advanced Education, Adelaide, South Australia, 1984 with major in education studies and minor in remedial English education. He got his certificate in secondary remedial education from Kuring-gai College of Advanced Education, Sydney, New South Wales, Australia, 1980 with major in English, English as a second language and mathematics. He got his diploma in teaching from Goulburn College of Advanced Education, New South Wales, Australia, 1978 , general primary with major in cultural studies.

Trevor's PhD research in the sociology of education, at UniSA, dealt with investigating the cultural and learning identities of socially-marginalized groups. Teaching extensively in government and independent schools in Australia and Hong Kong, as well as working within a university context, has enabled him to interact with disparate student populations (both internally and online) from a range of professional areas including: early childhood, primary, middle and upper secondary schooling, adult and workplace education. He has also studied theories related to differences and similarities in the cognition of learners in cross-cultural contexts; especially in relation to language use and learning styles. Trevor's master of education studies involved understanding communication within one's own socio-cultural group and how language can be taught across cultures. 